PESQUIMAT, Revista de la F.C.M. de la

Universidad Nacional Mayor de San Marcos

Vol. XI - Nº1 Pgs. 21-30 Lima - Perú Oct. 2008

\title{
CONTROLABILIDAD EXACTA INTERNA PARA LA ECUACIÓN SEMILINEAL DEL CALOR
}

\author{
Víctor Rafael Cabanillas Zannini ${ }^{1}$
}

Resumen.- El presente trabajo trata acerca del problema de la controlabilidad exacta interna para la ecuación semilineal del calor con no linealidad globalmente Lipschitz. Usando el Teorema sobreyectivo de Minty-Browder demostramos que la ecuación semilineal del calor es exactamente controlable en $L^{2}(\Omega)$. Este resultado fue obtenido por $W$. Liu y $G$. Williams [5] para no linealidades de la forma $f(y)$. Siguiendo las ideas expuestas en [5] probamos el mismo resultado de controlabilidad pero para no linealidades más generales involucrando términos gradientes $f(y, \nabla y)$.

Palabras claves: Controlabilidad exacta, ecuación semilineal del calor, Teorema de Minty-Browder, Método de la Energía.

\begin{abstract}
This work is concerned with the study of exact internal controllability problem for the semilinear heat equation with nonlinearities globally Lipschitz. Using the Minty-Browder surjective theorem we show that the semilinear heat equation is exactly controllable in $L^{2}(\Omega)$. For nonlinearities of the form $f(y)$, this result was obtained by $W$. Liu and G. Williams [5]. Following the ideas exposed in [5] we show the same controllability result but for more general nonlinearities involving gradient terms $f(y, \nabla y)$.
\end{abstract}

Key words: Exact controllability, semilinear heat equation, Minty-Browder Theorem, Energy Method.

\footnotetext{
${ }^{1}$ UNMSMS, Facultad de Ciencias Matemáticas. E-mail:vcabanillasz@unmsm.edu.pe
} 


\section{INTRODUCCION}

Sea $\Omega$ un conjunto abierto acotado de $\mathbb{R}^{N}, N \geq 1$, con frontera de clase $C^{2}$. Dado $T>0$, denotemos por $Q=\Omega \times(0, T)$ el cilindro generado por $\Omega$ y por $\Sigma=\partial \Omega \times(0, T)$ su frontera.

Consideremos la ecuación semilineal del calor

$$
\begin{cases}y^{\prime}-\Delta y+f(t, y, \nabla y)=h & , \text { en } Q \\ y=0 & \text {, sobre } \Sigma \\ y(0)=y^{0} & \text {, en } \Omega\end{cases}
$$

En relación a la función $f$ asumiremos la siguiente hipótesis:

(H) La función $f:[0, T] \times \mathbb{R} \times \mathbb{R}^{N} \longrightarrow \mathbb{R}, f=f(t, x, y)$. es continua en la variable temporal $t$ y globalmente Lipschitz en las variables $(x, y) \in \mathbb{R} \times \mathbb{R}^{N}$, con constante de Lipschitz $L>0$, es decir,

$$
\left|f\left(t, x_{1}, y_{1}\right)-f\left(t, x_{2}, y_{2}\right)\right| \leq L\left(\left|x_{1}-x_{2}\right|+\left|y_{1}-y_{2}\right|\right)
$$

En (1.1), $h=h(x, t)$ representa la función control. Por $y^{\prime}$ denotamos la derivada de la función $y$ con respecto a la variable temporal $t$. Decimos que el sistema (1.1) es exactamente controlable en $L^{2}(\Omega)$ en el tiempo $T>0$ si la siguiente condición es satisfecha:

Para cualquier $z^{0} \in L^{2}(\Omega)$ existe una función control $h \in L^{2}\left(0, T ; H^{-1}(\Omega)\right)$ tal que la solución y de (1.1) satisface:

$$
y(T)=z^{0}
$$

Es decir, un sistema como (1.1) es exactamente controlable sobre $L^{2}(\Omega)$ en el tiempo $T$ cuando, dado un estado final $z^{0} \in L^{2}(\Omega)$ es posible encontrar una función control que al actuar sobre el sistema conduzca al estado $u=u(\cdot, t)$ hacia el estado terminal $z^{0}$ en un tiempo $T$.

En los últimos años, la controlabilidad de sistemas parabólicos no lineales (semilineales, superlineales, fuertemente no lineales, etc.) ha sido intensamente estudiada. Una excelente referencia al respecto lo constituye el artículo de E. Zuazua [8]. La controlabilidad exacta para la ecuación lineal del calor en un tiempo arbitrario resulta imposible cuando el control actúa sobre un subconjunto propio del dominio $\Omega$, tal como fue probado por 
J. L. Lions en [4], de la misma forma resulta imposible alcanzar cualquier estado en un tiempo arbitrario en el caso semilineal. En el presente trabajo mostramos que para tiempos adecuados es posible lograr tal controlabilidad cuando la función control se encuentra distribuida sobre todo el dominio $\Omega$.

El artículo está organizado de la siguiente manera: en la sección 2 presentamos el principal resultado de este artículo; la sección 3 está dedicada a establecer nociones preliminares acerca de operadores monótonos y el Teorema de Minty-Browder. En la sección 4 recordamos algunos resultados de existencia y unicidad de soluciones para la ecuación semilinea del calor y construimos un operador no lineal F, además de probar su monotonicidad. Finalmente, en la sección 5 damos una idea de la demostración del resultado principal.

\section{EL RESULTADO PRINCIPAL}

El principal resultado de este artículo establece lo siguiente:

Teorema 2.4 Si la hipótesis $(\boldsymbol{H})$ es satisfecha, entonces existe un tiempo $T_{0}>0$ tal que para $0<T \leq T_{0}$, el sistema (1.1) es exactamente controlable en $L^{2}(\Omega)$ en el tiempo $T$, es decir, dado cualquier estado inicial $y^{0} \in L^{2}(\Omega)$ y cualquier estado terminal $z^{0} \in L^{2}(\Omega)$, existe una función control $h=h(x, t)=h\left(x, t, y^{0}, z^{0}\right) \in L^{2}\left(0, T ; H^{-1}(\Omega)\right)$ tal que la solución y de (1.1) satisface (1.2).

La principal herramienta para la demostración de este teorema lo constituye el Teorema de Minty-Browder. Este teorema y algunos preliminares adicionales son recordados en la siguiente sección. Debemos indicar además que el resultado es constructivo en el sentido que nos permite encontrar la función control $h$.

\section{PRELIMINARES}

Definición 3.1. Sea $X$ un espacio de Banach real. Por $X^{\prime}$ denotamos el espacio dual de $X$, y por $\langle\cdot, \cdot\rangle_{X^{\prime} \times X}$ la dualidad entre $X^{\prime}$ y $X$. Dado un operador $F: X \longrightarrow X^{\prime}$ entonces

(i) F es llamado monótono si y solamente si

$$
\langle F u-F v, u-v\rangle_{X^{\prime} \times X} \geq 0, \text { para todo } u, v \in X
$$


(ii) $F$ es llamado fuertemente monótono si y solamente si existe una constante $\lambda>0$ tal que

$$
\langle F u-F v, u-v\rangle_{X^{\prime} \times X} \geq \lambda\|u-v\|_{X}^{2}, \text { para todo } u, v \in X
$$

(iii) F es llamado hemicontinuo si y solamente si la aplicación

$$
t \longrightarrow\langle F(u+t v), w\rangle_{X^{\prime} \times X}
$$

es continua en $[0,1]$ para todo $u, v, w \in X$.

(iv) $F$ es llamado coercivo si y solamente si

$$
\lim _{\|u\|_{X} \longrightarrow \infty} \frac{\langle F u, u\rangle_{X^{\prime} \times X}}{\|u\|_{X}}=+\infty
$$

Mayores referencias y resultados relacionados con estas definiciones pueden ser encontrados en el libro de E. Zeidler [7].

Observación 3.1. Si $X=L^{2}(\Omega)$, entonces, gracias al Teorema de Representación de Riesz, es posible identificar el espacio $L^{2}(\Omega)$ con su dual, es decir, $L^{2}(\Omega) \equiv\left(L^{2}(\Omega)\right)^{\prime}$. En tal caso, un operador $F: L^{2}(\Omega) \longrightarrow L^{2}(\Omega)$ es fuertemente monótono si y solamente si existe una constante $\lambda>0$ tal que

$$
\int_{\Omega}(F u(x)-F v(x))(u(x)-v(x)) d x \geq \lambda\|u-v\|_{X}^{2}
$$

para todo $u, v \in L^{2}(\Omega)$.

Teorema 3.1 (Minty-Browder). Sea $X$ un espacio de Banach reflexivo, y $F: X \rightarrow X^{\prime}$ un operador monótono, hemicontinuo y coercivo. Entonces $F$ es un operador sobreyectivo. Además, si $F$ es fuertemente monótono, entonces existe el operador inverso $F^{-1}: X^{\prime} \rightarrow X$ y es Lipschitz continuo.

Demostración. Ver E. Zeidler [7]. 


\section{CONSTRUCCIÓN DE UN OPERADOR NO LINEAL}

En esta sección construiremos un operador no lineal $F: L^{2}(\Omega) \rightarrow L^{2}(\omega)$ y probaremos que es fuertemente monótono.

Consideremos el siguiente sistema retrógrado en el tiempo con estado terminal $u^{T}$

$$
\begin{cases}-u^{\prime}-\Delta u=0 & , \text { en } Q \\ u=0 & , \text { sobre } \Sigma \\ u(T)=u^{T} & , \text { en } \Omega\end{cases}
$$

Cuando $u^{T} \in L^{2}(\Omega)$, el sistema (4.1) tiene una única solución $u=u(x, t)$ en la clase

$$
u \in C\left([0, T] ; L^{2}(\Omega)\right) \cap L^{2}\left(0, T ; H_{0}^{1}(\Omega)\right)
$$

Si $u^{T} \in H^{2}(\Omega) \cap H_{0}^{1}(\Omega)$, existe una única solución $u$ de (4.1) en la clase

$$
u \in C\left([0, T] ; H^{2}(\Omega) \cap H_{0}^{1}(\Omega)\right) \cap L^{2}\left(0, T ; H_{0}^{1}(\Omega)\right)
$$

Ver [2].

Además, $\Delta$ es el generador infinitesimal de un semigrupo de contracciones de clase $C_{0}$ y un semigrupo analítico de operadores sobre $L^{2}(\Omega)$. (Ver [6]).

Cuando $u^{T} \in D(\Delta)=H^{2}(\Omega) \cap H_{0}^{1}(\Omega)$ tenemos $u-\Delta u \in C\left([0, T] ; L^{2}(\Omega)\right)$.

Entonces, el sistema

$$
\begin{cases}y^{\prime}-\Delta y+f(t, y, \nabla y)=u-\Delta u & , \text { en } Q \\ y=0 & \text {, sobre } \Sigma \\ y(0)=y^{0} & \text {, en } \Omega\end{cases}
$$

admite una única solución $y=y(x, t)$ tal que

$$
y \in C\left([0, T] ; L^{2}(\Omega)\right) \cap W\left(0, T ; H^{2}(\Omega) \cap H_{0}^{1}(\Omega), L^{2}(\Omega)\right)
$$

donde $W\left(0, T ; H^{2}(\Omega) \cap H_{0}^{1}(\Omega), L^{2}(\Omega)\right)$ es el espacio de Banach de las funciones $u \in L^{2}\left(0, T ; H^{2}(\Omega) \cap H_{0}^{1}(\Omega)\right)$ cuya derivada débil $u^{\prime}$ pertenece a $L^{2}\left(0, T ; L^{2}(\Omega)\right)$ y equipado con la norma 


$$
\|u\|_{W\left(0, T ; H^{2}(\Omega) \cap H_{0}^{1}(\Omega), L^{2}(\Omega)\right)}^{2}=\|u\|_{L^{2}\left(0, T ; H^{2}(\Omega) \cap H_{0}^{1}(\Omega)\right)}^{2}+\left\|u^{\prime}\right\|_{L^{2}\left(0, T ; L^{2}(\Omega)\right)}^{2}
$$

Ver [7] para los detalles.

Ahora, para un estado inicial $y^{0} \in L^{2}(\Omega)$ definimos el operador no lineal

$$
\begin{gathered}
F\left(y^{0}, \cdot\right): D(\Delta) \subset L^{2}(\Omega) \rightarrow L^{2}(\Omega) \\
F\left(y^{0}, u^{T}\right)=y(\cdot, T)
\end{gathered}
$$

Los siguientes dos lemas tienen el objetivo de colocar el operador $F$ en las condiciones del teorema sobreyectivo de Minty-Browder. Más precisamente, en los próximos lemas probaremos que $F$ es un operador continuo y fuertemente monótono.

Lema 4.1 Si la hipótesis (H) es válida, entonces el operador F definido en (4.6) es Lipschitz continuo.

Demostración. Sean $u_{1}$ y $u_{2}$ dos soluciones de (4.1) con estados finales $u_{1}^{T}, u_{2}^{T} \in D(\Delta)$, y sean $y_{1}$ y $y_{2}$ las soluciones de (4.5) correspondientes a $u_{1}, y_{1}^{0}$ y $u_{2}, y_{2}^{0}$. Entonces tenemos

$$
\begin{cases}\left(y_{2}-y_{1}\right)^{\prime}-\Delta\left(y_{2}-y_{1}\right)+ & \\ f\left(t, y_{2}, \nabla y_{2}\right)-f\left(t, y_{1}, \nabla y_{1}\right)=u_{2}-u_{1}-\Delta u_{2}+\Delta u_{1} & , \text { en } Q \\ y_{2}-y_{1}=0 & , \text { sobre } \Sigma \\ \left(y_{2}-y_{1}\right)(0)=y_{2}^{0}-y_{1}^{0} & , \text { en } \Omega\end{cases}
$$

Multiplicando (4.7) por $y_{2}-y_{1}$, integrando sobre $Q_{t}=\Omega \times(0, t)$ y aplicando técnicas de energía obtenemos

$$
\int_{\Omega}\left|y_{2}(t)-y_{1}(t)\right|^{2} d x \leq C\left[\left\|y_{2}^{0}-y_{1}^{0}\right\|_{L^{2}(\Omega)}^{2}+\left\|u_{2}^{T}-u_{1}^{T}\right\|_{L^{2}(\Omega)}^{2}\right]
$$

para cualquier $t \in[0, T]$. Si $t=T$ tenemos

$$
\left\|F\left(y_{2}^{0}, u_{2}^{T}\right)-F\left(y_{1}^{0}, u_{1}^{T}\right)\right\|_{L^{2}(\Omega)}^{2} \leq C\left[\left\|y_{2}^{0}-y_{1}^{0}\right\|_{L^{2}(\Omega)}^{2}+\left\|u_{2}^{T}-u_{1}^{T}\right\|_{L^{2}(\Omega)}^{2}\right]
$$

lo cual termina la prueba.

Observación 4.1 Por el Lema 3.1 y usando un argumento de densidad, el operador $F\left(y^{0}, \cdot\right)$ puede ser extendido a $L^{2}(\Omega)$. Usaremos la misma notación para tal extensión $F$, es decir, en adelante cuando hagamos referencia al operador $F$, nos estaremos refiriendo al operador definido sobre $L^{2}(\Omega) \times L^{2}(\Omega)$. 
Observación 4.2 Multiplicando la ecuación (4.1) por un factor integrante es posible demostrar que existe una constante $\delta>0$ tal que la siguiente estimativa de decaimiento para las soluciones de (4.1) es válida

$$
\int_{\Omega}|u(t)|^{2} d x \leq \exp (-\delta(T-t)) \int_{\Omega}|u(T)|^{2} d x
$$

para $t \in[0, T]$.

Lema 4.2 Asumamos la validez de la hipótesis $(\boldsymbol{H})$. Supongamos que L ó T es lo suficientemente pequeño de tal forma que satisfaga

$$
M(L, T)=1-\exp (-\delta T)-L^{2} \widetilde{C}>0
$$

donde $\delta$ es la constante encontrada en (4.8) y $\widetilde{C}$ es una constante positiva de inmersión. Entonces, para cualquier $y^{0} \in L^{2}(\Omega)$ fijo, el operador $F\left(y^{0}, \cdot\right): L^{2}(\Omega) \rightarrow L^{2}(\Omega)$ es fuertemente monótono.

Demostración. Primero demostramos para $u_{1}^{T}, u_{2}^{T} \in D(\Delta)$, y entonces, por un argumento de densidad, la demostración es válida para $u_{1}^{T}, u_{2}^{T} \in L^{2}(\Omega)$.

Multiplicando (4.7) por $u_{2}-u_{1}$, integrando sobre $Q$ y usando la estimativa de decaimiento exponencial para las soluciones de (4.1) obtenemos

$$
\int_{\Omega}\left[F\left(y^{0}, u_{2}^{T}\right)-F\left(y^{0}, u_{1}^{T}\right)\right]\left(u_{2}^{T}-u_{1}^{T}\right) \geq\left(1-\exp (-\delta T)-L^{2} \widetilde{C}\right)\left\|u_{2}^{T}-u_{1}^{T}\right\|_{L^{2}(\Omega)}^{2}
$$

es decir,

$$
\left(F\left(y^{0}, u_{2}^{T}\right)-F\left(y^{0}, u_{1}^{T}\right), u_{2}^{T}-u_{1}^{T}\right)_{L^{2}(\Omega)} \geq M(L, T)\left\|u_{2}^{T}-u_{1}^{T}\right\|_{L^{2}(\Omega)}^{2}
$$

como se deseaba.

\section{CONTROLABILIDAD EXACTA}

Siendo $F\left(y^{0}, \cdot\right): L^{2}(\Omega) \rightarrow L^{2}(\Omega)$ un operador fuertemente monótono, dado $z^{0} \in L^{2}(\Omega)$, el Teorema de Minty-Browder implica que existe $u^{T} \in L^{2}(\Omega)$ tal que $F\left(y^{0}, u^{T}\right)=z^{0}$, es decir, $y(T)=z^{0}$. En consecuencia, el sistema (4.4) es exactamente controlable en $L^{2}(\Omega)$ con control $h=u-\Delta u \in L^{2}\left(0, T ; H^{-1}(\Omega)\right)$, siendo $u$ la solución de (4.1). 
Notemos sin embargo, que la condición (4.9) en el Lema 3.2 es una condición muy restrictiva. Si $L$ es una constante de Lipschitz tal que $M(L, T)<0$ no existe controlabilidad exacta. Para salvar esta deficiencia, introducimos, como en [5], el Método de la Expansión del Dominio.

Bosquejo de la demostración del Teorema 1.1. Con el fin de demostrar el resultado de controlabilidad exacta para cualquier constante de Lipschitz $L$, introducimos para $\tau>0$ el dominio expandido

$$
\Omega_{\tau}=\tau \Omega=\{\tau ; x \in \Omega\}
$$

y los conjuntos

$$
Q_{1, \tau}=\Omega_{\tau} \times(0,1), \quad \Sigma_{1, \tau}=\partial \Omega_{\tau} \times(0,1)=\{(\tau x, t) ;(x, t) \in \Gamma \times(0,1)\}
$$

Definimos una función $f_{\tau}$ por

$$
f_{\tau}(t, u, \nabla u)=\frac{1}{\tau^{2}} f\left(\frac{t}{\tau^{2}}, u, \nabla u\right)
$$

Procediendo como en [5], en lugar de (4.1) y (4.4) consideramos los sistemas

$$
\begin{cases}-u^{\prime}-\Delta u=0 & , \text { en } Q_{1, \tau} \\ u=0 & , \text { sobre } \Sigma_{1, \tau} \\ u(x, 1)=u^{1}(x) & , \Omega_{\tau}\end{cases}
$$

y

$$
\begin{cases}w^{\prime}-\Delta w+f_{\tau}(t, w, \nabla w)=u-\Delta u & , \text { en } Q_{1, \tau} \\ w=0 & , \text { sobre } \Sigma_{1, \tau} \\ w(x, 0)=w^{0}(x) & , \Omega_{\tau}\end{cases}
$$

Entonces, el operador $F$ definido por (4.6) se convierte en

$$
F\left(w^{0}, u^{1}\right)=w(\cdot, 1)
$$

Sea $\tau_{0}$ tal que $\tau_{0}^{4}-\tau_{0}^{4} \exp (-\delta)-L^{2} \widetilde{C}>0$, entonces $\tau^{4}-\tau^{4} \exp (-\delta)-L^{2} \widetilde{C}>0$ para $\tau \geq \tau_{0}$. En consecuencia, de los lemas 3.1 y 3.2 sigue que $F$ es un operador continuo y fuertemente monótono sobre $L^{2}\left(\Omega_{\tau}\right)$. Entonces, aplicando el Teorema Minty-Browder concluimos que para cualquier $z^{0} \in L^{2}(\Omega)$ existe una función $u^{1} \in L^{2}\left(\Omega_{\tau}\right)$ tal que 


$$
F\left(w^{0}, u^{1}\right)=v^{0}
$$

donde

$$
v^{0}(x)=z^{0}\left(\frac{x}{\tau}\right), x \in \Omega_{\tau}
$$

Entonces, resolvemos (5.1) con estado terminal $u^{1}$. Esto permite hallar la función control

$$
u-\Delta u \in L^{2}\left(0, T ; H^{-1}(\Omega)\right)
$$

tal que la solución de (5.2) satisface

$$
w(x, 1)=v^{0}, x \in \Omega_{\tau}
$$

Haciendo

$$
\begin{gathered}
y(x, t)=w\left(\tau x, \tau^{2} t\right), x \in \Omega, t \geq 0 \\
h\left(x, t ; y^{0}, z^{0}\right)=\tau^{2}\left[u\left(\tau x, \tau^{2} t\right)-\Delta u\left(\tau x, \tau^{2} t\right)\right]
\end{gathered}
$$

Vemos que $y$ satisface (1.1) y (1.2).

Entonces, hemos demostrado que para $0<T=\frac{1}{\tau^{2}} \leq \frac{1}{\tau_{0}^{2}}=T_{0}$ el sistema (1.1) es exactamente controlable en $L^{2}(\Omega)$ en el tiempo $T$.

Agradecimiento. El autor agradece al Profesor L. A. Medeiros por habernos sugerido el problema de este artículo, así como por las diversas discusiones que tuvimos al respecto. 


\section{BIBLIOGRAFÍA}

[1] R. Dautray-J. L. Lions, Functional and Variational Methods in Mathematical Analysis and Numerical Methods for Science and Technology, Vol. 2. Springer-Verlag, Berlin, 1992.

[2] R. Dautray-J. L. Lions, Evolution Problems in Mathematical Analysis and Numerical Methods for Science and Technology, Vol. 5. Springer-Verlag, Berlin, 1992.

[3] J. L. Lions, Contrôlabilité exacte, stabilisation et perturbations de systèmes distribués. Vol 1, 2, Masson, RMA 8\&9, Paris, 1988.

[4] J. L. Lions, Remarques sur la contrôlabilité approchée, Jornadas Hispano-Francesas sobre Control de Sistemas Distribuidos, Universidad de Málaga, p.7787, 1991.

[5] W. Liu - G.Williams, Exact Internal Controllability for the Semilinear Heat Equation, J. Mat. Anal. and Appl., 211, 258-272, 1997.

[6] A. Pazy, Semigroups of Linear Operators and Applications to Partial Differential Equations. Springer-Verlag, New York, 1983.

[7] E. Zeidler, Nonlinear Functional Analysis, Vol. II/A and II/B, Springer-Verlag, New York, 1990.

[8] E. Zuazua, Controllability of Partial Differential Equations and its Semi-Discrete Approximations, Discrete and Continuous Dynamical Systems, Vol 8, No 2, p.469513, 2002. 